\title{
Risk scoring systems in colorectal surgery-future factors
}

\author{
Aninda Chandra • Tarun Singhal • Biju Aravind • \\ Catherine Bryant $\cdot$ Sudhakar Mangam
}

Accepted: 13 May 2009/Published online: 30 May 2009

(C) Springer-Verlag 2009

Dear Editors:

RE: Skala K, Gervaz P, Buchs N, Inan I, Secic M, Mugnier-Konrad B, Morel P. Risk factors for mortalitymorbidity after emergency-urgent colorectal surgery. Int $\mathbf{J}$ Colorectal Dis 24:311-6, 2009.

There are a number of scoring systems which exist, but they often have a large data burden or are hard to calculate, and the authors have tried to explore the risk factors in emergency-urgent colorectal (bowel resection) surgery. The authors are to be commended in their aspirations and in aiming to determine risk factors to be used in future surgical scoring systems. This prospective 5-year study promised much yet requires clarification from the authors. Could the authors elucidate why they chose patients undergoing resection of bowel rather than that outlined in the title (all emergent-urgent colorectal surgery)?

Overall, Table 3 details six factors that were significant. The authors do not discuss age which may be a proxy for physiological reserve and a surrogate marker for undeclared comorbidity or the wide inter-observer variability of the ASA physical status score. In 1996, Hall and Hall found that if the ASA score was III or more and the age was over 60 years, this identified over $80 \%$ of the patients who died or had significant morbidity (prolonged stay in hospital, developed intraperitoneal sepsis or were admitted to the

A. Chandra $\cdot$ T. Singhal $\cdot$ B. Aravind $\cdot$ C. Bryant Department of Colorectal and General Surgery,

Princess Royal University Hospital,

Farnborough, London, UK

S. Mangam

Department of Colorectal Surgery,

Queen Elizabeth Queen Mother University Hospital,

Margate, Kent, UK

A. Chandra $(\bowtie)$

58 Hopwood Gardens, Tunbridge Wells, Kent TN4 9PU, UK

e-mail: aninda_chandra@hotmail.com
ICU). As the ASA is subjective, the importance of consultant anaesthetic assessment should be highlighted, and this was not mentioned in the study. Blood loss, particularly as reported by the operating surgeon, is potentially subjective; is this why the authors used $500 \mathrm{ml}$ as a cutoff value? The data may have been more informative if categorised or subdivided more appropriately; blood loss and transfusion may have been more informative if subdivided, and should those who are overweight (body mass index, BMI $=25-29$ ) be considered in the same category as those who are obese (BMI $>30)$ ? Further, if those with an ASA of 4-5 (17\% of patients) were excluded, how would this have affected the results?

Rather than POSSUM which the authors cite, many authors use P-POSSUM which overcomes this criticism. The benefits of P-POSSUM include a lower baseline prediction of $0.2 \%$, whilst linear individual mortality and also morbidity can be predicted. Neither of these scoring systems were designed to look at emergency-urgent surgery alone. Gawande et al. in 2008 reported an objective intraoperative surgical APGAR score derived from 303 patients and prospectively validated in 767 patients. Gawande found that intraoperative blood loss rather than perioperative blood loss was an important risk factor. Skala et al. are to be commended in realising the importance of blood loss independently. The surgical APGAR score also incorporates two other factors which were lowest mean arterial pressure and lowest heart rate (or arrhythmias) to develop a ten-point model, neither of which seems to be included in the data but could as easily have been measured. Overall, the authors have analysed a study with much data that strengthen that of Gawande et al.; however, one wonders what else might have been revealed.

Yours faithfully,

Aninda Chandra, Tarun Singhal, Biju Aravind, Catherine Bryant, Sudhakar Mangam 